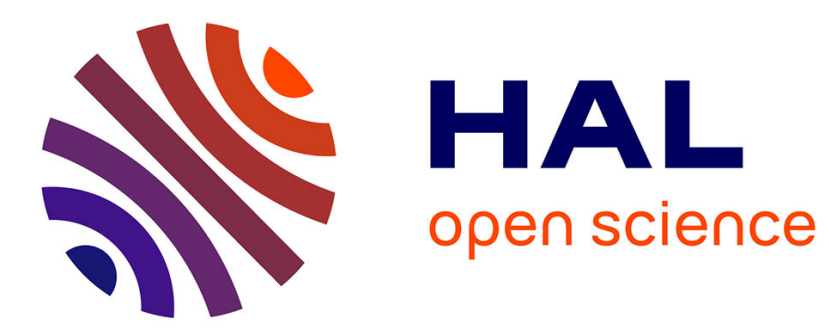

\title{
Des objets et leurs musées : en guise d'introduction
} Michèle Coquet

\section{To cite this version:}

Michèle Coquet. Des objets et leurs musées : en guise d'introduction. Journal des Africanistes, 1999, 69 (1), pp.7 - 28. 10.3406/jafr.1999.1184 . hal-03433094

\section{HAL Id: hal-03433094 https://hal.science/hal-03433094}

Submitted on 17 Nov 2021

HAL is a multi-disciplinary open access archive for the deposit and dissemination of scientific research documents, whether they are published or not. The documents may come from teaching and research institutions in France or abroad, or from public or private research centers.
L'archive ouverte pluridisciplinaire HAL, est destinée au dépôt et à la diffusion de documents scientifiques de niveau recherche, publiés ou non, émanant des établissements d'enseignement et de recherche français ou étrangers, des laboratoires publics ou privés. 


\section{Des objets et leurs musées : en guise d'introduction}

Michèle Coquet

Citer ce document / Cite this document :

Coquet Michèle. Des objets et leurs musées : en guise d'introduction. In: Journal des africanistes, 1999 , tome 69, fascicule 1. Des objets et leurs musées. pp. 7-28;

doi : https://doi.org/10.3406/jafr.1999.1184

https://www.persee.fr/doc/jafr_0399-0346_1999_num_69_1_1184

Fichier pdf généré le 09/05/2018 


\section{Des objets et leurs musées}


Michèle COQUET ${ }^{*}$

\section{Des objets et leurs musées : en guise d'introduction}

«L'esprit n'est tenu en éveil et vivement sollicité par le besoin de se développer en présence des objets qu'autant qu'il reste en eux quelque chose de mystérieux qui n'a pas encore été révélé.»

André BRETON, L'amour fou.

Pour avoir fréquenté les réserves de plusieurs grands (par la taille et l'ancienneté) musées d'ethnographie européens, je me suis souvent demandé si toutes ces vieilles choses amassées, poussiéreuses et malodorantes parfois, ne poursuivaient pas ceux qui avaient la charge de veiller sur elles d'une vindicte comparable, toutes proportions gardées (je ne connais pas de mort mystérieuse de conservateur), à celle de la momie de Toutankhamon. Le conservateur des collections africaines de l'un de ces prestigieux établissements m'accompagna un jour dans les sous-sols pour me montrer des objets d'Afrique centrale et orientale. Après les avoir fait glisser péniblement, il ouvrit les portes de lourdes armoires de métal semblables à des coffres-forts sur de délicats trésors de paille, de plumes, de perles et de cuir tressé : chapeaux de notables kuba, costumes de masques, poupées, tabliers et ceintures de femmes himba. Je lui fis remarquer comme un effet d'émiettement, ici et là : petits débris végétaux, fragments de cuir épars et chapeaux grignotés. "Ce sont les souris », m'expliqua-t-il d'un air las. Mais les armoires « coffres-forts »? Il leva les bras dans un geste d'impuissance. Dans un autre de ces meubles, étaient alignés d'étranges paquets noirs, de forme

* Chargée de recherches, URA 221 du CNRS, 27 rue Paul Bert, 94204 Ivry-sur-Seine cedex. 
oblongue, aux flancs troués. «Ah! fis-je, les souris ? - Non! Ça, c'est le conservateur ; pendant la guerre, il n'avait plus de tabac !»

En racontant cette anecdote, je ne veux en aucun cas remettre en cause les compétences et la qualité du travail de mon hôte. Elle me paraît, seulement, illustrer assez justement, sur le ton de la fable, les difficultés à la fois pratiques et intellectuelles auxquelles nous confrontent les objets ethnographiques et les institutions en charge de les conserver. Elle est également une manière d'ouverture à ce texte, et au recueil qu'il annonce. Différents thèmes y sont abordés : histoires de la collecte et de la formation du musée d'ethnographie en tant que genre, fonctions contemporaines du Musée, nature et définition de l'objet «ethnographique », l'objet témoin de civilisation, questions de scénographie et d'exposition. Cette réflexion est menée depuis une dizaine d'années par nos collègues américains et anglais, mais demeure pour l'instant peu développée en France. Ce numéro du Journal participe, modestement, au débat ; et la perspective de la création prochaine d'un Musée des arts et des civilisations en rappelle l'urgence.

\section{ÉLÉMENTS D'HISTOIRE}

Le musée d'ethnographie, ou des civilisations, est l'héritier d'une longue tradition qui aurait débuté sous le signe de la collection privée, qu'elle ait été du sanctuaire ou de l'église, du roi, du noble ou du chef militaire. Cette tradition ne nous est pas propre; dans d'autres sociétés du monde, et en toute époque, les hommes ont collectionné. La plus ancienne, et sans doute la plus émouvante, remonte au Moustérien. Son initiateur serait cet homme de Néanderthal que bien des musées, de préhistoire cette fois, ont coutume de nous présenter le front bas, l'œil sauvage, et la bouche lippue sur le menton fuyant. L'homme, malgré ses apparences, a fait montre d'une curiosité esthétique certaine, peut-être même d'un émerveillement, comme le souligne André Leroi-Gourhan en décrivant cette petite moisson, découverte à Arcy-sur-Cure, qui comprend «deux masses de pyrite de fer formées de sphères rugueuses agglomérées, le moule interne d'une grosse coquille de gastéropode fossile et un polypier sphérique de l'ère secondaire" (LeroiGourhan 1965 : 213). L'auteur ajoute : «C'est le premier témoin attesté de la reconnaissance de formes [...] le premier signe [...] de la quête du fantastique naturel [...] forme d'adolescence des sciences naturelles car dans toutes les civilisations l'aurore scientifique débute dans le bric-à-brac des "curios" " (Ibid. : 213-214. cf. également le commentaire de Pomian 1987 : 40-42). Ute Röschenthaler rappelle plus loin l'importance des trésors des grands chefs camerounais du Grassland, composés d'objets hétéroclites, dont certains de 
provenance européenne, comme des roues de bicyclette, obtenus par échange, achat ou tribut, et dont l'ampleur témoigne, aux yeux de tous, de la grandeur et du pouvoir de leur propriétaire. Aujourd'hui, l'Afrique villageoise compte de nombreux «collectionneurs », non pas d'objets rares et chers, mais, plus simplement, d'objets de culture. À travers ce geste de conservation, ils ne revendiquent pas la pratique elle-même, qui n'a pas d'existence en tant que telle, mais un statut social, celui de fonctionnaire ou de notable, et un rôle, né par un effet de boomerang de l'attitude occidentale face à ces mêmes objets, de revendications identitaires et de la défense des valeurs traditionnelles. Je connais des paysans enrichis au Burkina Faso qui ornent les murs de leur maison au toit de tôle (un autre signe de distinction sociale), non pas d'objets décoratifs européens, mais de copies de masques grandeur nature ou miniaturisés, et de tissus traditionnels ${ }^{1}$. L'histoire des musées a donné lieu à de nombreuses publications. Parmi les ancêtres du musée d'ethnographie, on trouve le cabinet de curiosités, à la mode durant les $\mathrm{XVI}^{\mathrm{e}}$ et $\mathrm{XVII}^{\mathrm{e}}$ siècles, et les collections royales et princières comportant tant des objets exotiques que des livres ou des antiquités. À l'origine de la constitution de ces collections, il y a non seulement la volonté de prestige des classes nobles ou bourgeoises de la société européenne, mais aussi un désir de connaissance du passé, des sciences et des arts. Le musée moderne naît en France au XVIII ${ }^{e}$ siècle avec la Révolution. Il n'est plus le produit de curiosités et d'initiatives personnelles, mais devient un instrument de gestion étatique du patrimoine au profit de la société civile. Sa vocation pédagogique et identitaire se construira, se consolidera tout au long du $\mathrm{XIX}^{\mathrm{e}}$ siècle, et cette fonction prendra un tour particulier avec les musées d'ethnographie pendant la période coloniale : « l'institution muséale [...] renforce sa mission d'éducation [...] elle aide les peuples d'Europe à prendre conscience de leur identité » (Rivière 1989 : 52). Les musées d'ethnographie, lors de leurs créations au XIX ${ }^{\mathfrak{e}}$ siècle, intègrent souvent les muséums d'histoire naturelle, en général plus anciens (celui de Paris est fondé en 1793), et rejoignent «curios», et spécimens de la faune et de la flore, surtout exotiques. C'est le cas du Pitt Rivers Museum à Oxford ou de l'American Museum of Natural History à New York, et de nombreux autres musées américains.

Les muséums d'histoire naturelle et les musées d'ethnographie du XIX ${ }^{\mathrm{e}}$ siècle apparaissent à nos yeux plus proches du cabinet de curiosités que ce que nous pensons devoir être des musées de sciences, même si les principes ayant présidé à leur conception relèvent de présupposés scientifiques et

1 Sur le même sujet, et à propos du Grassland, voir Fowler, 1997. 
ethnologiques. La juxtaposition, sinon le mélange, d'éléments provenant de la faune et de la flore avec des artefacts que n'unissent pas toujours des liens géographiques ou temporels, ou le regroupement des objets dans une contemporanéité scénographique niant la différence des provenances et des époques, tout cela évoque la disposition du cabinet de curiosités, qui rassemblait dans une même totalité le fossile, le bézoard et le fétiche à clous. L'effet de collage nous apparaît évident mais il est sans doute dans la destinée du musée d'ethnographie de le rendre plus manifeste qu'il ne l'est dans les musées d'art classique. L'apparente hétérogénéité produite par le spectacle des œuvres, offertes d'ordinaire au regard du visiteur en autant de compositions improbables - disparité des formes, des matériaux, des compositions, des tailles, des usages, etc. - contribue aussi à cet effet «cabinet de curiosités ", que l'on ne rencontre pas dans un musée d'art classique où ne se côtoient que la sculpture, plutôt de marbre et de bronze, et la peinture de chevalet sur toile (pour l'art contemporain, la situation apparaît différente). Ce n'est sans doute pas un hasard si les œuvres choisies pour le Pavillon des sessions au Louvre relèvent toutes de la sculpture. L'homogénéité ainsi construite réorganise un corpus d'œuvres non occidentales dans une perspective scénographique classique, et écarte l'effet de chaos ou de désordre, l'effet «cabinet de curiosités », que peut produire une vitrine de musée de civilisation, où le regroupement des objets paraît souvent le fait du hasard. Mais autre chose encore entretiendrait de manière implicite cette filiation avec la Kunst- und Wunderkammer, la "chambre des merveilles»: les ensembles d'objets entreposés au musée constituent les «œuvres» singulières de collecteurs et d'ethnographes, essentiellement de la seconde moitié du XIX siècle et du premier quart du $\mathrm{XX}^{\mathrm{e}}$ siècle. Les collections actuelles reposent sur cet acquis précolonial, rarement évoqué explicitement dans les musées eux-mêmes, et c'est particulièrement vrai, en Europe, pour les collections africaines. La recherche ethnographique de la première heure s'est appuyée sur la collecte d'objets pour jeter les bases de ses théories: une étape nécessaire comme le rappelle le petit manuel intitulé Instructions sommaires pour les collecteurs d'objets ethnographiques publié en 1931 par le musée d'Ethnographie du Trocadéro. Si les conditions difficiles de voyage et la brièveté de séjour en un même lieu ne permettaient pas d'acquérir une connaissance approfondie des populations rencontrées, en revanche le ramassage de grandes quantités d'objets au $\mathrm{XIX}^{\mathrm{c}}$ siècle donnait l'illusion de pallier cette impuissance, et la collecte, en tant qu'activité propre, devenait le processus même de constitution d'un savoir : étiqueter, classifier, juger, attribuer, formèrent autant d'opérations transformant l'information en 
connaissance, chaque artefact fournissant une réponse à une question qui n'avait pu être posée.

\section{LE MUSÉE ET LA COLLECTION}

Une collection se constitue à la suite de choix et de refus, au terme d'une sélection où, indépendamment de tout critère scientifique, le goût personnel, celui de l'époque, le statut social du collecteur, l'étendue de son savoir, etc., jouent un rôle non négligeable. L'exemple des collecteurs du XIX ${ }^{\mathrm{e}}$ siècle mandatés par les musées (je ne parle pas ici des libres voyageurs) est instructif : ils entendent non seulement participer à l'élaboration d'une science naissante, l'anthropologie, mais encore, pour William H. Sheppard, missionnaire noir américain, œuvrer à la reconnaissance des droits de l'Africain et du Noir américain, ou pour Herbert Lang et James P. Chapin, envoyés par l'American Museum of Natural History de New York, et pour Emil Torday, collectant pour le British Museum, de démontrer l'existence du sentiment esthétique et de réalisations relevant de l'art parmi les peuples africains, au prix, parfois, de quelques distorsions à la réalité (cf. The Scramble for Art in Central Africa). Les attitudes sur le terrain sont ambiguës : tandis que H. Lang et J.-P. Chapin favorisent l'essor d'un art mangbetu figuratif proche des canons européens, avec pour motif iconographique distinctif la coiffure féminine évasée prolongeant le crâne déformé, E. Torday refuse d'acquérir un masque kuba comportant des matériaux européens (tissus et perles de traite) car il témoigne d'influences exogènes à la culture kuba. Des styles ethniques et des traditions sont inventés à partir de l'idée d'une Afrique non contaminée par l'Occident, refermée sur elle-même, où un objet, comme toute autre donnée du savoir, pour être authentique, doit apparaître rare et affranchi de toute influence reconnaissable. Tous ces présupposés eurent pour conséquence la constitution de collections très incomplètes - c'est inhérent à leur nature - et surtout fondées sur la subjectivité ; certains objets étaient collectés mais pas d'autres. Ces histoires, celles des collectes, de leurs circonstances et des principes idéologiques et scientifiques qui les conduisent, habitent les objets ethnographiques ( $c f$. infra l'article de Ute Röschenthaler). Ceux-ci sont des « voyageurs », comme l'écrit J. Clifford (1996 : 191-192), souvent depuis longtemps, ici comme là-bas. Constituées d'une somme d'aventures particulières, dont les souvenirs sont exposés dans les vitrines, ces histoires encombrent la «mémoire » des musées d'ethnographie. Elles composent à leur tour une histoire, en partie occultée, ou volontairement oubliée, que le musée concrétise de manière exemplaire, en tant que puissant lieu d'exposition de notre propre culture, à travers le regard qu'elle porte sur 
les autres, pensées nécessairement basées sur le mode de la «grande » différence. Une histoire sociale, politique, et même esthétique : tout le monde sait combien l'image que nous a renvoyée le miroir déformant des arts dits «primitifs» a compté dans l'élaboration de notre esthétique et de nos pratiques artistiques durant tout le $\mathrm{XX}^{\mathrm{e}}$ siècle. Les collecteurs, les musées d'ethnographie, les grandes expositions organisées à la fin du XIX ${ }^{\mathrm{e}}$ siècle $^{2}$, ont joué une part active dans la formation du goût des sociétés européennes, soit que ces arts aient dispensé aux nôtres, sur le mode d'une lente infusion avant le bouleversement cubiste, leurs formes, leurs couleurs, les traits déroutants de leurs figures humaines, soit qu'ils aient été à l'origine de créations marquées par l'idéologie coloniale. Dans le premier cas, la découverte et l'étude en ces mêmes dernières années du $\mathrm{XIX}^{\mathrm{e}}$, par des théoriciens de l'art comme Owen Jones, Aloïs Riegl ou Henry Balfour, de ces autres modes d'expression, en particulier de leur élément décoratif, ont contribué à leur reconnaissance. Quant à la seconde évocation, je l'illustrerai par un exemple. Parmi les objets rapportés du continent africain en quantité considérable au $\mathrm{XIX}^{\mathrm{e}}$ siècle, figurent les armes, lances, couteaux de jet, poignards, etc. qui occupent pêle-mêle et par milliers les réserves des musées. Elles étaient certainement plus aisément transportables, et accumulables, que d'autres artefacts, ce qui explique en partie leur abondance; on peut se demander tout de même si la disposition, propre à cette période de la fin du $\mathrm{XIX}^{\mathrm{e}}$ et du début du $\mathrm{XX}^{\mathrm{e}}$ siècle, à chérir l'ornement, n'a pas été déterminante. Nombre de ces armes, comme les lances luba ou les couteaux de jet, comportaient, en effet, de riches décors stylisés ou offraient par leur morphologie même, une plastique répondant au penchant de l'époque. Une fois accrochées aux parois des musées et des maisons particulières, ces armes servaient des objectifs ornementaux, en tant qu'éléments décoratifs d'assemblages ordonnés, reprenant parfois l'image du «trophée » antique. Le musée d'ethnographie devenait le lieu d'expression d'un art ornemental, héraut de la perfection de l'équilibre symétrique dont les éléments constitutifs étaient des artefacts sans identité ; l'ornement ne revendique pas d'autre référent en effet que le support sur lequel il se trouve. Ces quelques traits de caractère qui définissent le musée d'ethnographie importunent.

Le musée d'ethnographie, et des civilisations non-occidentales sans doute plus que tout autre, ne peut être qu'un lieu de conservation imparfait pour des objets en matériaux souvent fragiles, rarement conçus pour survivre

\footnotetext{
2 Des expositions importantes d'objets africains ont été organisées par le musée de Leipzig en 1892, à Anvers en 1894 et à Bruxelles en 1897. La première exposition commerciale d'art primitif s'est déroulée à Paris, en 1919 (Goldwater 1988 : 25-28).
} 
à leur utilisateur initial, élaborés parfois seulement pour les quelques heures de leur exhibition rituelle, et qui subissent davantage, par là-même, la marque du temps. Il ne peut être qu'un lieu de représentation également imparfait, car quelques dizaines ou centaines d'objets kanak, kwakiutl ou dogon ne sauraient décrire les civilisations kanak, kwakiutl ou dogon; et même si un musée, éventualité improbable, pouvait recueillir la totalité des objets produits par une culture, celle-ci serait-elle mieux représentée pour autant? La diversité de ces objets, leur identité parfois inconnue et souvent incertaine, leur histoire, non pas celle des transactions qui les ont portés jusqu'à nous mais celle de leur conception et de leur utilisation, une histoire pour une grande part à jamais perdue - et quoi de plus pesant que l'oubli ? -, sont autant de données qui rendent plus que difficile la tâche de ceux qui les étudient. L'ensemble de ces données, auquel il convient d'ajouter celles que l'on a précédemment mentionnées, constituant le lot commun de tous les musées d'ethnographie, a contribué, en France en particulier, à provoquer la méfiance des ethnologues : ces institutions leur sont apparues comme des lieux de perdition, des lieux où se seraient échoués des objets épaves, produits de collectes nécessairement partielles et partiales, effectuées parfois dans des conditions douteuses, dont on ne connaîtrait plus de leur origine qu'un nom d'ethnie. L'objet ethnographique appartiendrait de fait davantage au passé de son collecteur (institution, ethnographe ou voyageur) qu'à celui de son propriétaire initial. On mesurera ici l'ampleur de l'aporie sur laquelle semble reposer le musée d'ethnographie; l'essentiel de ses collections, issues de sociétés qui ne connaissent pas ou peu l'écriture, regarde davantage vers le passé que vers le présent et l'avenir, vers un passé qui n'est pas le nôtre, et qui, pour une grande part, nous échappe. Tout musée, selon la définition de Jean-Louis Déotte, et j'ajouterai, plus problématiquement le musée d'ethnographie, dissocie l'objet de son lieu, de ce pour quoi et pour qui il a été fabriqué et conçu, de sa «mémoire destinale » (Déotte 1996:36); il est un espace de «suspension », une «institution d'oubli actif», une «friche » (Idem). En conséquence, le musée se doit d'être aussi un lieu de lutte et de vigilance, active, contre l'oubli; les utilisateurs et les concepteurs de ces objets, ou leurs descendants, tels que les Amérindiens, mais aussi les Africains, comme le démontre ici Salia Malé, sont aujourd'hui engagés dans cette tâche en se réappropriant ces institutions et leur contenu.

\section{L'OBJET AU MUSÉE}

Détérioration éventuelle, identité lacunaire, écho assourdi d'un passé opaque, autant d'images évoquées par l'objet ethnographique au sein du 
musée, objet qui apparaît souvent, pour la communauté des ethnologues, comme le membre détaché d'un corps disparu. La définition de l'objet ethnographique telle qu'elle avait été posée lors de la constitution des collections du musée de l'Homme, en 1937, par Paul Rivet et Georges Henri Rivière, était celle de l' «objet-témoin », de l' " échantillon », du «spécimen de civilisation », de l'« idéogramme matériel » (ou de la «condensation des représentations collectives ») nous renseignant sur la technologie, la culture matérielle (la pêche, la chasse, l'agriculture, la forge, etc.), les croyances et les rites. Comme l'a très bien analysé Jean Jamin dont je reprends ici les termes (Jamin 1996), cette attitude, qui présentait à l'époque la nouveauté d'abolir les distinctions entre arts mineurs et arts majeurs jusque-là en cours, relevait d'une «positivité par excès » (Jamin 1985: 61) : l'idée d'échantillonnage répondait à la nécessité de démontrer que l'ethnologie était une science qui utilisait des méthodes taxinomiques comparables aux autres. Elle reposait aussi sur un modèle de collecte et de muséologie idéales tel que Marcel Mauss l'avait pensé : « le collecteur s'attachera à composer des séries logiques, en réunissant si possible tous les échantillons d'un même objet en dimensions, formes, etc., sans craindre les doubles et les triples » (Mauss 1967: 17). Tout ethnologue familier des conditions de travail qu'offre le terrain mesurera la difficulté de l'entreprise. Nous avons admis depuis lors que le modèle de classification employé pour les œuvres de la nature ne s'accordait pas aux productions humaines : l'objet ethnographique ne peut être considéré ni comme un échantillon - il ne donne une idée précise ni de l'ensemble des objets fabriqués par une culture, ni de la culture elle-même, car il n'illustre rien et n'est pas un symbole -, ni comme témoin, si ce n'est comme témoin muet et souvent énigmatique 3 .

La question que pose, et que posera toujours, le musée d'ethnographie, est celle de la saisie de l'objet hors de sa réalité ethnographique et de la restitution possible de celle-ci, sous d'autres formes et en d'autres lieux, lourd fardeau qui donne également du souci aux ethnologues : ces derniers, à défaut d'objets mais dans une perspective identique, bataillent avec des mots. Leur attitude face à cette institution découle d'une autre constatation et pose une autre question. L'objet, bien loin d'être susceptible de devenir un échantillon - et le devenir un échantillon présuppose que l'opération qui consiste à extraire l'objet de son contexte ne l'ampute pas, pour reprendre la métaphore corporelle déjà employée, d'une partie de sa signification - , serait en fait indissociable de la réalité polymorphe, insaisissable et profondément vivante

${ }^{3}$ Pour de plus amples commentaires sur la question de l'objet-témoin, je renvoie à l'analyse de J. Jamin dans l'article cité précédemment. 
dans laquelle et pour laquelle il est conçu. Cette constatation vient de la pratique d'un métier dont la vocation est l'étude de sociétés vivantes et de tous les faits que l'on peut y observer. Le vivant ne se détacherait-t-il pas irrémédiablement de l'objet dès lors que celui-ci est collecté ? D'où le recours, dans les expositions, à ces écrans où la vidéo doit rendre le mouvement à ce qui n'en a plus. Piètre subterfuge, et, je pense, erreur de conception muséographique... Où est l'homme dans l'objet ? Avec l'objet ethnographique échantillon, la question avait été, en un sens, éludée. L'objet était alors regardé davantage sous l'angle de sa fonction (alimentaire, agricole, guerrière, religieuse, etc.) ou de sa valeur (de prestige, d'usage, de pouvoir, etc.). Cette interrogation - où est l'homme dans l'objet? - procède d'une attitude qui voudrait que l'objet soit étudié, et ne fasse pleinement sens, que lorsqu'il est en pleine action. Je reprends là les termes de Arnold Van Gennep dont la position était encore plus catégorique : « [...] notre vrai domaine d'étude, c'est le mécanisme de la production même, c'est-à-dire de la fabrication technique et des conditions psychiques et sociales sous-jacentes dont les objets ne sont que des témoins desséchés, comme les plantes d'un herbier » (Van Gennep 1985 : 49). Afin de rendre compte, de la manière la plus fidèle possible, de cette dimension de l'objet saisi sur le vif, Marcel Mauss proposait dans son Manuel d'ethnographie, toujours dans une perspective de conservation idéale, de procéder à une description très détaillée des objets : chacun devait être accompagné d'une fiche "donnant les renseignements sur l'usage et la fabrication, accompagnée d'une annexe photographique et cinématographique », d'un dessin «chaque fois qu'il faudra montrer le maniement de l'objet », notant le type d'utilisateur, les moments de son utilisation, les raisons, religieuses ou magiques, la signification des décorations (Mauss 1967 : 17). Les intentions étaient bonnes. Mais le travail de description a rarement pu être fait avec cette précision, et quand bien même il l'aurait été, pouvait-il satisfaire à cette exigence qui veut que l'objet ne fasse sens qu'en pleine action? Car la saisie recommandée par Marcel Mauss est celle de l'instant ethnographique qui ne peut englober les transformations et les modifications à venir que provoque nécessairement l'usage.

L'objet auquel est confronté l'ethnologue occupe une place particulière en ce qu'il se trouve à la charnière de la vie sociale et de la vie rituelle et religieuse. Les matériaux qui le composent renseignent sur l'environnement naturel ou, par exemple, sur les relations commerciales qui ont permis son obtention. Sa facture, comme pour tout objet, témoigne d'un certain savoir-faire, d'une réflexion sur les formes, les matières, les couleurs, etc., l'esthétique. Médiateur entre des entités surnaturelles et les hommes, il est souvent au cœur des rites. Sa transmission et sa manipulation peuvent 
mobiliser le groupe social (on pensera ici à la kula ou au potlatch). Voici les faits qu'étudie une anthropologie de l'objet, un objet situé au centre de la relation qui unit l'homme au monde et au groupe auquel il appartient. L'objet que rencontre l'ethnologue serait comparable à l'arbre qui cache la forêt : derrière lui, se profile toute une société en mouvement, bruissante de mots prononcés, de gestes et de pensées accomplis. La tradition du musée d'ethnographie veut, suivant la perspective ethnologique, et de manière paradoxale puisque les ethnologues ne s'y reconnaissent que rarement, que les objets soient présentés comme des sortes de supports visuels et matériels, comparables à des balises, nécessaires à la compréhension des systèmes de pensée des cultures d'où ils proviennent ; ils accompagnent la découverte, par le visiteur d'une exposition, de la société au sein de laquelle ils ont été conçus. Ils seront peut-être relayés, voire dominés dans le futur, par d'autres approches de la réalité ethnographique tels que les médias interactifs. Les ethnologues sont en effet les inspirateurs de ce point de vue, dans la mesure où ils ont donné la priorité dans les analyses où l'objet était évoqué à l'étude des contextes - de fabrication, d'utilisation, de manipulation. Cependant, si l'objet renvoie à l'ensemble des représentations d'une culture, il n'en est jamais l'illustration ou la transcription. Il constitue un mode d'expression qui a ses règles spécifiques de génération et de signification, et s'inscrit toujours dans un système, non seulement celui des relations qui le lie à ses utilisateurs, mais aussi celui d'un réseau de formes, de matières, de figures, ou d'images ; il peut être considéré comme un morceau de pensée matérialisée ou objectivée, par ses formes, ses matériaux, sa texture, sa consistance, sa structure, son décor. Si l'étude du contexte socioculturel constitue le préalable nécessaire et indispensable, elle a pris bien souvent le pas sur une réflexion centrée sur les objets eux-mêmes. Le musée d'ethnographie doit devenir le lieu de cette réflexion qui permettra de gérer de manière vivante les collections existantes, et c'est à ce prix que les objets retrouveront cette partie substantielle d'eux-mêmes qui les rendra de nouveau riches de sens. Mais quelle pourrait être cette réflexion portant sur les objets eux-mêmes et intéressant tant la muséographie que l'anthropologie?

Un objet ne fait sens qu'à l'intérieur de l'ensemble des relations qui l'unit, non seulement à ceux qui l'utilisent mais aussi aux autres objets avec lesquels il est utilisé, et par rapport auxquels il est conçu, tant d'un point de vue fonctionnel que morphologique et intellectuel. Cependant, le réseau de ces relations apparaît comme couvrant une étendue aux frontières floues, ce qu'évoque avec une grande justesse la réflexion de B. Kirshenblatt-Gimblett rapportée ici par Brigitte Derlon : «Où l'objet commence-t-il et où finit-il ? [...] Devons-nous exposer la tasse avec la soucoupe, le thé, la crème et le 
sucre, la cuillère, la nappe et le napperon, la table, la chaise, la carpette ? » Cette question sur la définition de l'objet en tant qu'élément d'un ensemble plus vaste, un objet indissociablement lié, dans le monde moderne, à son utilisation en tant qu'objet d'exposition et de musée - tout objet, aujourd'hui, est exposable ou susceptible, un jour, de le devenir et d'être dissocié, pour reprendre le terme de Jean-Louis Déotte, de sa mémoire destinale -, a été posée dans certaines ceuvres d'art contemporain: Edward Kienholz, par exemple, exposa dans les années 1960 et 1970 des environnements d'objets, reprenant des scénographies du quotidien. L'un d'entre eux, propriété du musée d'Art moderne du Centre Georges Pompidou, propose aux visiteurs le spectacle - du kitsch pur - d'une chambre à coucher baignée de rose avec bibelots, lampes, chaises, fauteuils, etc. et radio diffusant une musique grésillante et désuète ${ }^{4}$. L'œuvre d' $E$. Kienholz s'inscrit dans les préoccupations artistiques de cette seconde moitié de siècle, qui ont profondément modifié le regard porté sur l'objet, non plus perçu comme une concrétude close sur elle-même, mais comme une présence prolongée par l'espace environnant et les autres éléments, êtres vivants et choses inertes, qui 1'habitent. Dans une telle scénographie - qui fait recours à des objets de civilisation -, aucun objet n'est particulièrement valorisé, et il s'agit bien plutôt (entre autres) de la mise en scène, à la fois d'un mode de relations entre objets différents, et d'un ensemble dont on construit ainsi la cohérence et qui fait sens en tant que tel, mais qui n'est pas fermé sur lui-même, ce que suggère la musique distillée par la radio, ouvrant sur le monde extérieur. Cette démarche artistique, et l'interrogation qu'elle révèle, sont toujours actuelles comme l'atteste l'œuvre récente de l'artiste russe Ilya Kabakov, qui expose la série de pièces d'un intérieur soviétique, traces d'usage à l'appui (matelas crevé, affiches de propagande déchirées, crasse et saleté, cela aussi faisant partie intégrante de l'objet global, l'œuvre) ${ }^{5}$. La réflexion qui a accompagné la création des éco-musées repousse encore les limites du « système d'objets » et est parvenue à des conclusions audacieuses à travers la redéfinition de la notion de patrimoine: les «objets », de civilisation, de l'éco-musée, comprennent également les paysages, essentiellement ruraux, dont «la conservation ne peut être séparée d'un usage et d'une transformation par ceux qui les utilisent et y vivent ». L'objet de musée dans ce cas «conserve des liens avec le monde » (Davallon 1996 : 74). La redéfinition de la notion de

${ }^{4}$ L'œuvre s'intitule Tandis que des visions de prunes confites dansent dans leurs têtes et date de 1976.

5 Dans C'est ici que nous vivons, exposition organisée par le Centre Georges Pompidou du 17 mai au 4 septembre 1995. 
patrimoine fait alors basculer le "patrimoine d'objet (un "trésor" déjà amoncelé) en un patrimoine en projet», passant ainsi «d'un patrimoine réalisé à un patrimoine virtuel » (Idem: 75). «Le patrimoine est non seulement fait de choses du passé ou de la nature, mais [...] il englobe aussi les êtres vivants » (Idem: 76). L'objet ethnographique serait bien cet arbre qui cache la forêt, une forêt vue non seulement sous l'angle d'un contexte, comme cela était évoqué auparavant, mais aussi sous celui, à son tour, d' « objet » de musée. On rencontre un écho de ces idées de saisie globale dans les vieux dioramas des muséums d'histoire naturelle de la fin du $\mathrm{XIX}^{\mathrm{e}}$ et de la première moitié du $\mathrm{XX}^{\mathrm{e}}$ siècle, que Franck Beuvier décrit ici, et dont les exemplaires concernant l'Afrique, conservés à l'American Museum of Natural History, font figures de modèle ( $c f$. Vogel 1988). Au premier plan des mannequins grandeur nature s'affairent, parfois auprès d'animaux empaillés, vêtus, parés, et usant d'artefacts - les objets de musée - devant la porte de leur demeure ou parmi les troncs de la sylve équatoriale. Le second et le troisième plans sont peints sur la toile du fond, le troisième plan se confondant avec un horizon qui ouvre sur l'infini, dans la meilleure tradition picturale de la Renaissance. Le regard vague de l'objet au paysage et à la ligne d'horizon ; l'artefact participe à un ensemble plus vaste qui le subsume, où le réseau le liant aux êtres et aux choses semble s'étendre jusqu'à se dissoudre.

\section{VERS UNE HISTOIRE DES FORMES, DES IDÉES ET DES ESTHÉTIQUES}

La réalité ethnographique se pose comme un ensemble foisonnant de relations à l'intérieur desquelles les objets tiennent une part des rôles en jeu ; les artefacts constituent en effet des matérialités confrontées à d'autres, les corps de ceux pour qui et par qui ils existent. Ils portent la trace des doigts qui les ont façonnés et utilisés, et ce, souvent de manière très visible : celle de l'usure, du polissage, des offrandes sacrificielles, de la patine, là où la main a laissé son empreinte à force de toucher. Ces traces énoncent les événements propres à l'histoire particulière de l'objet et aux manières de son usage ; en ce sens, tout objet, à peine a-t-il commencé à être, est le lieu de fixation du souvenir de ces manipulations. Les gestes de la fabrication, les erreurs, les remords, les décisions et les hésitations transparaissent. Aussi des présences de corps affleurent-elles à la surface de tout objet, gisant au cœur de sa masse, et qui font sens différemment selon la relation qui nous lie à lui. Les objets collectés durant les périodes précoloniales impliquent également, de manière particulière, le corps de leurs collecteurs et ethnographes : lorsque ceux-ci retournèrent en Europe, ces objets constituèrent la preuve tangible, à la fois de leur séjour en ces terres lointaines, avec tout ce qu'il comprenait de dangers 
et d'incertitudes, et des relations, bonnes ou mauvaises, créées, au quotidien, avec les autochtones (Fabian 1998: 81).

Système d'objets, présence de corps dans les objets, sont autant de questions posées à l'anthropologue et au muséographe quant au statut ontologique de l'objet. Par ailleurs, l'anthropologie s'intéresse, institutionnellement, mais avec un engagement inégal, à certaines modalités d' «être et de faire sens» dans les objets. L'anthropologie des techniques s'est préoccupée de ces présences de corps en étudiant la production des objets, les gestes et les savoir-faire mis en œuvre pour leur élaboration, les rapports sociaux sollicités ou provoqués par leur conception, et leur matérialité, c'est-à-dire leur structure et le type de travail opéré sur le matériau. Ce domaine de l'anthropologie a bénéficié d'un fondateur de poids en la personne de André Leroi-Gourhan, lui-même précédé par Marcel Mauss. Les approches iconologique et esthétique contribuent également à cette recherche anthropologique portant sur les objets eux-mêmes, deux voies de recherche n'ayant pas eu le même succès en France que l'anthropologie des techniques, en partie à cause de l'importance, dans la constitution de l'anthropologie culturelle, de la tradition sociologique issue des travaux de Émile Durkheim et de Marcel Mauss, qui a négligé les champs de l'iconologie et de l'esthétique. Ces deux approches doivent redonner à l'objet, qu'il soit ou non empreint d'une valeur artistique, la place qui lui revient dans sa culture. L'approche iconologique, à partir de l'étude des codes plastiques et stylistiques, et des thèmes iconographiques, permet d'envisager, corrélativement, l'histoire des formes et des idées, et de démontrer comment les objets constituent des modes de signification spécifiques et autonomes. À propos du style, à savoir cette logique qui préside à la combinaison des formes et des matières, André Leroi-Gourhan rappelait qu'il était révélateur de certaines dispositions esthétiques et intellectuelles : «l'Orientaliste retrouvera un clou chinois dans un tas de mille clous (de tous les coins du monde). Et si un clou chante chinois, comme un poème de la dynastie des T'ang, on ne voit pas pourquoi on n'essayerait pas de traduire son chant» (Leroi-Gourhan 1953 : 71-72). À ce propos, l'ouvrage Documents pour l'art comparé de l'Eurasie septentrionale (1948) est exemplaire parce que conçu à partir de collections de musées. Il s'agit d'un essai d'iconologie portant sur des décors d'objets usuels lapons, cuillers et couteaux en particulier, en bois de renne gravé, et sur les thèmes rencontrés dans les broderies finnoises, dénommés thèmes des « deux chevaux », des « oiseaux » et de l' « arbre aux oiseaux », et du «personnage debout ». Dans ce recueil de courtes analyses, André Leroi-Gourhan se livre à une étude comparative, portant sur des séries, des transformations morphologiques de motifs figurant ces différents thèmes, transformations 
s'effectuant à la fois dans l'espace (les territoires eurasiens) et dans le temps, puisque certains thèmes se retrouvent sur des objets appartenant à des sociétés anciennes, comme ceux de l'arbre aux oiseaux ou du couple rapace-herbivore fréquemment représenté en Mésopotamie. Il tente de donner les règles de la «diffusion et du choix des thèmes » ainsi que celle de l' "évolution morphologique » des motifs, se présentant sous une forme figurative, ou géométrique et fortement stylisée. Mais sa motivation principale demeure celle d' " évaluer l'aide que l'art populaire actuel ou récent peut apporter à l'histoire de l'art des grands peuples, et rechercher si des séries actuelles sur un même thème éclairent les variations morphologiques des documents isolés qu'étudie l'archéologie » (Idem: 88).

En s'intéressant à «l'évolution historique des thèmes » et en regardant du côté de l'archéologie, André Leroi-Gourhan introduisait les dimensions chronologiques et historiques dans un domaine, celui de l'étude des expressions plastiques des sociétés traditionnelles, où elles sont rarement invoquées : une attitude un peu paresseuse a prévalu jusqu'ici, qui considère que, le poids de la tradition ayant tendance à freiner toute innovation dans ces cultures, les œuvres quelles qu'elles soient échappent aussi, en grande partie, au changement. L'approche iconologique peut pallier cette grave lacune en réintroduisant l'histoire dans l'étude des objets ethnographiques. Sally Price, dans un ouvrage très roboratif consacré au regard occidental sur les arts des sociétés exotiques, rappelle que des ethnologues comme Franz Boas avaient, en leur temps, préconisé « une plus grande attention à la création, l'innovation et le changement historique » (1995:93). Elle-même a insisté, dans ses études touchant à l'art des Saramaka du Surinam, sur l'importance de cette dimension historique : elle démontre que les Saramaka en ont conscience dans l'élaboration de leur art, et qu'ils en parlent. « Les Saramaka, écrit-elle, distinguent et dénomment quatre styles de sculpture sur bois, et les situent précisément dans le temps, non par datation en termes d'années numérotées, mais en liaison avec des personnages et événements contemporains pour lesquels ils connaissent les rapports chronologiques. De même, ils sont conscients de changements importants dans leur art sur calebasse (par exemple le passage du décor externe au décor interne ou le transfert concurrent de ce moyen d'expression des hommes aux femmes). Ils connaissent et expliquent aussi très bien l'évolution de leur art du textile, qui est passé successivement de la broderie libre à une sorte de patchwork à petits carrés, à des compositions à base de longues bandes, enfin à un style élaboré de broderie au point de croix » (Price 1995 : 168).

L'exemple saramaka nous décrit la place que tient l'histoire dans toute culture, même si elle est de tradition orale, et comment les objets peuvent en 
conserver les traces à travers les transformations et les emprunts stylistiques ou thématiques. Il nous rappelle qu'il est grand temps que soit conduite une véritable histoire de l'art, au sens large du terme, des sociétés exotiques, dites sans écriture, histoire qui n'existe pas, ou presque pas, et qui est en général, encore balbutiante. Une véritable histoire de l'art prend en compte le contexte socioculturel, ce qui nécessite une connaissance anthropologique des cultures; c'est là que vient s'inscrire une anthropologie de l'objet telle qu'elle a été définie précédemment. Elle considère à la fois les propriétés plastiques et stylistiques des auvres et leur iconographie et doit s'effectuer dans une double perspective, comparative et chronologique. Il est significatif de constater qu'aujourd'hui les masques africains, pour ne parler que de ceux-là, si abondamment représentés dans les musées et dans les collections privées, dont l'usage est très répandu dans les cultures d'Afrique subsaharienne, qui appartiennent plus que tout autre objet ethnographique à la catégorie si difficilement définissable du « chef-d'œuvre », comptent, en proportion, parmi les objets auxquels très peu d'études ont été consacrées. La majeure partie des sociétés de l'aire mandé-voltaïque, installées dans la partie soudanaise de l'Afrique occidentale, ont recours aux masques dans leurs rites. Si l'on connaît, bien que de manière encore insuffisante, leur rôle dans la vie sociale (dans les initiations ou les sociétés secrètes par exemple) et dans la vie rituelle et religieuse (ils célèbrent, en particulier, par leurs sorties périodiques les cycles agraires et des saisons, et les cycles initiatiques), que sait-on de ce qu'ils énoncent à travers leurs formes, les matériaux utilisés, les figures, les gestes de la danse ? Pourquoi tant d'animaux sont-ils représentés ? Que nous révèlent ces images des idées exprimées sur la matière, la nature, l'art, les hommes, les dieux, etc. ? Pourquoi tant d'ingéniosité dans la diversité des formes? Que connaît-on des idées, des grands thèmes qui y sont inscrits ? Que sait-on des artistes et des artisans qui les ont fabriqués, de leurs conceptions artistiques, des influences du passé, des innovations et des emprunts? Rien, ou presque rien. Et qui dit emprunt, dit diffusion des modèles plastiques et des idées qui les accompagnent. C'est, entre autres, à ces conditions que les cultures demeurent vivantes, et les objets, même d'apparence humble, jouent dans ce processus un rôle substantiel. Cette grave ignorance est d'autant plus regrettable si l'on regarde l'histoire de l'art européen depuis un siècle : les sociétés non occidentales ou sans écriture y sont présentes, des peintures et des sculptures de Gauguin aux igloos de Mario Merz ou aux performances de Joseph Beuys. Le paradoxe inquiétant - mais est-ce si paradoxal ? - veut que, si nous avons intégré ces cultures à notre histoire de l'art, nous ne connaissons que bien peu de choses de la leur. L'histoire de l'art de ces sociétés est certes malaisée à conduire, les principaux 
documents que nous ayons, très fragmentaires, étant justement ceux que nous offrent les collections des musées d'ethnographie (et celles des particuliers), les archives coloniales, et la recherche sur le terrain, ce que démontre parfaitement l'exemple saramaka.

Ressaisir ces artefacts dans le cadre de l'histoire implique de reconsidérer la question de l'innovation, et par là-même de la création. L'innovation, en effet, existe et dans des proportions plus importantes qu'on ne pourrait le croire. Elle suit seulement des règles et des procédés qui ne nous sont pas familiers, ou qui sont moins visibles pour un œil mal averti. F. Boas avait changé l'angle d'approche en plaçant, non plus l'objet, mais l'artiste sur le devant de la scène, et avait souligné l'importance pour la connaissance des arts non occidentaux d'être attentif au point de vue des autochtones sur le processus même de la création, compris non seulement comme relevant d'un ensemble de techniques mais comme sollicitant également une réflexion artistique et esthétique. Ses étudiants ont suivi cette voie, et l'on peut sans doute attribuer à ces choix intellectuels et scientifiques le fait qu'il faille aujourd'hui se tourner vers les États-Unis pour trouver des travaux sur les arts exotiques qui relèvent de l'histoire de l'art et de l'esthétique. Il est intéressant à ce sujet de noter que Marcel Mauss, dans le chapitre que son Manuel d'ethnographie consacre à l'esthétique, n'accorde qu'une place restreinte au créateur de l'objet loin derrière l'esthétique, laquelle est envisagée avant tout comme un phénomène social. Les artisans et les artistes, qu'ils soient d'ici ou d'ailleurs, ont imprimé et impriment encore aujourd'hui à leurs œuvres un style personnel enseigné dans leurs « écoles », qu'ils dirigent en véritables maîtres d'ateliers, et repris par leurs apprentis. Bien sûr, cette histoire-là aussi est à faire. Par ailleurs, nous ne savons presque rien des conceptions esthétiques des créateurs et des utilisateurs des objets que nous avons dans les vitrines de nos musées. Certains impératifs propres à l'ethnologie ou à l'anthropologie privilégiant une vision globale de la société où l'individu n'occupe souvent de place que comme le reflet du groupe dans son ensemble, ont certainement joué, comme dans le cas de l'anonymat supposé des artistes autochtones. Or l'esthétique nécessite que l'on s'intéresse au sujet sentant et percevant, homme de la rue, artisan ou artiste. Elle exige que l'on prête attention à l'expression par la langue des subtilités de la compréhension esthétique du monde. Encore faut-il alors qu'il ne s'agisse pas seulement d'établir des lexiques, comme il en existe quelques-uns, renvoyant de manière implicite à une vision platonicienne de la beauté et de la laideur. Les conceptions de la beauté et de la laideur sont spécifiques à chaque culture et renvoient à des images et des expériences différentes, dont un lexique ne permet pas de rendre compte. Il faut donc nécessairement replacer chaque 
esthétique dans son cadre conceptuel, et l'approche esthétique ne considère pas les seuls objets mais s'attache aussi aux autres domaines d'expression de la culture : la musique, la poésie, le chant, la danse, la parure, le paysage, les «manières de table », etc. Elle permet également de comprendre comment les objets font sens pour ceux qui les créent, les regardent, les manipulent. Il convient d'ailleurs de rappeler que l'approche esthétique traite aussi de l'objet en pleine action, comme l'ont démontré certaines études portant sur des conceptions à l'œuvre dans le rite et relevant de catégories esthétiques (cf. Morphy 1992 et Jeudy-Ballini 1999). Et puis, il arrive fréquemment que ceux qui créent et conçoivent les objets (ou d'autres types d'œuvres, on pensera aux musiciens, souvent également chanteurs et poètes) développent, plus que les autres, une réflexion esthétique et un vocabulaire spécifique pour l'exprimer.

Ce voyage dans les différentes réalités ethnographique et muséographique fréquentées par l'artefact n'en aura pas épuisé la complexité. Tâche au demeurant impossible, puisque la destinée de l'objet est d'être pris dans un jeu continu, et vertigineux, d'interprétations, de gloses et d'hypothèses. Même dans son état originel, il ne fait pas sens de la même manière pour son auteur, son propriétaire, l'initié, le non initié, le simple passant, celui qui le vend et celui qui l'achète, l'autochtone et l'étranger, etc., et l'on pourrait livrer à l'infini d'autres situations encore. Philip Ravenhill, dans un ouvrage sur des sculptures baoulé nommées blolo, évoque ces jeux de réinterprétation à propos de la statuaire «colon»: le terme fut employé à l'origine par les Européens et les marchands d'art ivoirien et appliqué à des sculptures qui figurent pour chaque homme et pour chaque femme, dans la tradition baoulé, son partenaire de l'au-delà. Lorsque les sculpteurs attribuèrent à leurs figurines masculines des vêtements occidentaux, elles devinrent des «colons », témoignant, pour les Européens, de l'irrésistible humour africain. Les Africains les regardèrent comme des emblèmes de la triste période coloniale ; depuis, les sculpteurs qui perpétuent la fabrication et le style de ces objets pour les vendre, surtout aux touristes, décrivent leur travail comme relevant de la reconstitution historique et du maintien de traces du passé de la nation ivoirienne. La statue «colon» est bien devenue un objet souvenir, mais chacun se réserve le sien (Ravenhill $1996: 62-81$ ). Dès lors qu'il est sorti des mains de son créateur, l'objet est libre. C'est ce qui nous enchante, et c'est sur cette note que je voudrais finir. Le musée d'ethnographie, ou des civilisations, demeure, en tant que lieu, et si l'on se prête au jeu, un extraordinaire espace de capture, la nôtre. Car à l'appropriation raisonnée que nous nous proposons d'en faire, et à laquelle, justement, nous souhaitons associer les représentants des cultures concernées, l'objet oppose la présence 
irréductible de sa matérialité, la mémoire d'une intentionnalité lointaine, dans l'espace et souvent aussi dans le temps, qui, pour être résiduelle, n'en est pas moins là ; avec la force - et je me permets de reprendre, en leur donnant une autre tournure, quelques belles expressions que Claude Macherel nous offre dans son texte - qu'ont les « formes menues », « insidieuse ». Voilà l'enchantement.

\section{Bibliographie}

BALlÉE, Catherine (1996), «Le public: un enjeu des musées contemporains », Analyser le musée, Actes du colloque international organisé par l'Association suisse de sémiotique (ASS/SGS), Lausanne 21-22 avril 1995 : 49-67.

CLIFFORD, James (1996), Malaise dans la culture-L'ethnographie, la littérature et l'art au XX siècle, Paris, École nationale supérieure des Beaux-Arts (édition américaine : 1988).

Davallon, Jean (1996), "Réflexions sur "l'objet de musée" », Analyser le musée, Actes du colloque international organisé par l'Association suisse de sémiotique (ASS/SGS), Lausanne 21-22 avril 1995: 69-90.

DÉOTTE, Jean-Louis (1996), «Le musée, lieu de mémoire ? », Analyser le musée, Actes du colloque international organisé par l'Association suisse de sémiotique (ASS/SGS), Lausanne 21-22 avril 1995 : 33-67.

FABIAN, Johannes (1998), « Curios and Curiosity : Notes on Reading Torday and Frobenius », The Scramble for Art in Central Africa: 79-108.

FOWLER, Ian (1997), « Tribal and Palatine Arts of the Cameroon Grassfields : Elements for a "Traditional" Regional Identity ", in MacClANCY, Jeremy, Contesting Art-Art, Politics and Identity in the Modern World, Ethnicity and Identity Series, Oxford-New York, Berg : 63-84.

GOLDWATER, Robert (1988), Le primitivisme dans l'art moderne, Paris, PUF (édition américaine : 1938).

JAMIN, Jean (1996), «Introduction à Miroir de l'Afrique », in JAMIN, Jean, en collaboration avec J. MERCIER, Michel Leiris-Miroir de l'Afrique, Paris, Quarto, Gallimard.

- (1985), «Les objets ethnographiques sont-ils des choses perdues? », in HAINARD Jacques \& Roland KAEHR, Temps perdu-Temps retrouvé, musée d'Ethnographie de Neuchâtel.

Jeudy-Ballini, Monique (1999), «Dédommager le désir », Terrain, 32, mars : 5-20.

LeROI-GOURHAN, André (1948), Documents pour l'art comparé de l'Eurasie septentrionale, Paris, Les Éditions d'art et d'histoire.

- (1953), «Ethnologie et esthétique », Le Disque Vert 1 : 70-73. 
- (1965), Le geste et la parole. La mémoire et les rythmes, Paris, Albin Michel.

Mauss, Marcel (1967), Manuel d'ethnographie, Paris, Petite Bibliothèque Payot (première édition : 1947).

MorPHY, Howard (1999), «From Dull to Brilliant: The Aesthetics of Spiritual Power among the Yolngu », in COOTE, Jeremy \& Anthony Shelton, Anthropology, Art and Aesthetics, Oxford, Clarendon Press : 181-208.

PomIAN, Krzysztof (1987), Collectionneurs, amateurs et curieux. Paris, Venise : $X V I^{e}-X V I I I^{e}$ siècle, Paris, NRF, Gallimard.

PRICE, Sally (1995), Arts primitifs ; regards civilisés, Paris, École nationale supérieure des Beaux-Arts (édition américaine : 1989).

RAVENHILl, Philip L. (1996), Dreams and Reverie-Images of Otherworld Mates among the Baoule, West Africa, Washington and London, Smithsonian Institution Press.

Rivière, Georges-Henri (1989), «Musée et société, à travers le temps et l'espace », in La Muséologie selon G.-H. Rivière, Paris, Dunod.

The Scramble for Art in Central Africa, (1998), Edited by SCHILDKROUT, Enid \& Curtis A. KEIM, Cambridge, Cambridge University Press.

VAN GENNEP, Arnold (1985), «Quelques lacunes de l'ethnographie actuelle », in HAINARD, Jacques \& Roland KAEHR, Temps perdu-Temps retrouvé, musée d'Ethnographie de Neuchâtel.

Vogel, S. (éd.) (1989), Art / artifact. African Art in Anthropology Collections, New-York, The Center for African Art \& Prestel-Verlag. 